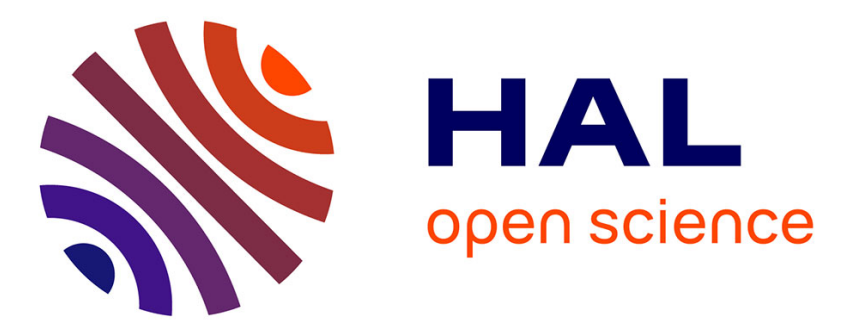

\title{
Physically and geometrically non-linear vibrations of thin rectangular plates
}

Ivan Breslavsky, Marco Amabili, Mathias Legrand

\section{To cite this version:}

Ivan Breslavsky, Marco Amabili, Mathias Legrand. Physically and geometrically non-linear vibrations of thin rectangular plates. International Journal of Non-Linear Mechanics, 2013, 58, pp.30-40. 10.1016/j.ijnonlinmec.2013.08.009 . hal-00864370

\section{HAL Id: hal-00864370 \\ https://hal.science/hal-00864370}

Submitted on 26 Feb 2015

HAL is a multi-disciplinary open access archive for the deposit and dissemination of scientific research documents, whether they are published or not. The documents may come from teaching and research institutions in France or abroad, or from public or private research centers.
L'archive ouverte pluridisciplinaire HAL, est destinée au dépôt et à la diffusion de documents scientifiques de niveau recherche, publiés ou non, émanant des établissements d'enseignement et de recherche français ou étrangers, des laboratoires publics ou privés.

\section{다(1)(2)}

Distributed under a Creative Commons Attribution - ShareAlikel 4.0 International 


\title{
Physically and Geometrically Non-linear Vibrations of Thin Rectangular Plates
}

\author{
Ivan Breslavsky, Marco Amabili*, Mathias Legrand
}

\begin{abstract}
Static deflection as well as free and forced large-amplitude vibrations of thin rectangular rubber plates under uniformly distributed pressure are investigated. Both physical, through a neo-Hookean constitutive law to describe the non-linear elastic deformation of the material, and geometrical non-linearities are accounted for. The deflections of a thin initially flat plate are described by the von Kármán non-linear plate theory. A method for building a local model, which approximates the plate behavior around a deformed configuration, is proposed. This local model takes the form of a system of ordinary differential equations with quadratic and cubic non-linearities. The corresponding results are compared to the exact solution and are found to be accurate. Two models reflecting both physical and geometrical non-linearities and geometrical non-linearities only are compared. It is found that the sensitivity of the deflection to the physically induced non-linearities at moderate strains is significant.
\end{abstract}

\section{Keywords}

neo-Hookean law — plate theory — non-linear vibrations — physical non-linearity — geometrical nonlinearity - hyperelasticity

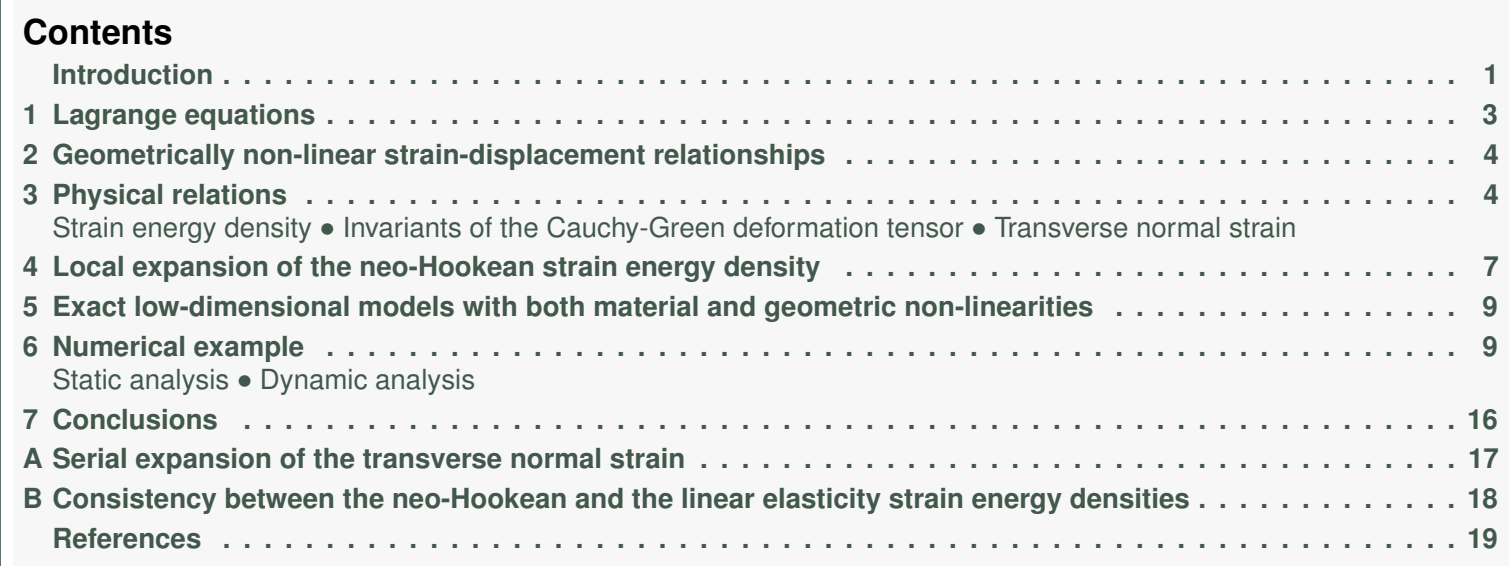

McGill University, Department of Mechanical Engineering, Macdonald Engineering Building, Room 270, 817 Sherbrooke Street West Montreal, Quebec, Canada H3A 0C3

${ }^{*}$ Corresponding author: marco.amabili@mcgill.ca

\section{Introduction}

Shell-type structures are widely used in engineering and are frequently subjected to periodic external loadings. In the vast majority of applications, classical materials like steels are considered. Nevertheless, in modern bio-engineering for instance, numerical predictions require the simulation of biological materials involving more sophisticated properties. Not only the geometrical non-linearity (the non-linearity of strain-displacements relations), but also the physical or material non-linearity (non-linearity of stress-strain relations) should be addressed. Reviews on geometrically non-linear vibrations of shells and plates can be found in [11, 26, 27, 3].

Several models describing the physical non-linearity aspect are available. A simple phenomenological model was developed by Kauderer, yet ignoring the framework of the widely accepted strain energy formulation [23]. This model is suitable for the description of non-linear elasticity of some metals, like copper for instance, and recent associated applications for dynamical problems of shelltype structures can be found in [6,8]. Nevertheless, models of hyperelasticity are preferred [23] for the description of rubber-like materials and soft biological tissues. However, due to the complicated nature 
of the corresponding derivations, only a few studies on the dynamics of shell and plate structures made of hyperelastic materials are reported $[15,16]$. A majority of these studies deal with simple geometries (spherical and circular cylindrical shells, circular membranes) and assume that the shape after deformation is identical. This allows for the derivation of closed-form expressions.

The first investigation on the radial oscillations of circular cylindrical tubes was undertaken by Knowles [20,21]. He considered tubes made of isotropic incompressible hyperelastic material and reduced the equations of motion to second order ordinary differential equations. In the same line, the paper by Akkas [1] deals with large-amplitude normal oscillations of a spherical membrane subjected to a uniform inflating step-pressure. It is found that a dynamic snap-out instability problem can occur for some values of the material coefficients. The relationship between the static behavior of the membrane and the dynamic instability is discussed; below the dynamic snap-out pressure threshold, the motion of the spherical membrane is always periodic. Finite-amplitude vibrations of non-linearly elastic incompressible spherical and circular cylindrical shells are mathematically examined by Calderer [10]. Due to the considered geometry it is assumed that the deformed structures are still a spherical or circular cylindrical shell, respectively. Under this assumption the radial periodic vibrations are investigated. Verron et al. [31] explored the dynamic inflation of spherical membranes of Mooney-Rivlin material for which a maximum in the frequency of oscillation versus pressure is detected.

Akyuz and Ertepinar [2] investigated cylindrical shells subjected to traction. Unlike other existing works in the literature, hyperelasticity is implemented through a Levinson-Burgess polynomial compressible material: this model is very similar to neo-Hookean one and coincides with it for incompressible materials. The static equilibrium in the finite deformation framework is first investigated, in the vicinity of which small-amplitude free vibrations are explored through small perturbation theory. The governing equations are solved numerically using a shooting method and the associated results compare well with the finite element solution. The condition of existence of periodic vibrations with respect to the geometrical and material shell properties is obtained.

Ref. [18] introduces an approximate non-linear ordinary differential equation for the displacement due to a sudden external pressure of an incompressible spherical membrane made of hyperelastic material modeled with the Ogden law. Below a critical pressure for which structural failure is observed, the dynamic response of the membrane is a non-linear periodic oscillation. In the same spirit, the work of Ren [28] determines the critical suddenly exerted pressure for an incompressible neo-Hookean tube. Below the critical pressure, the fixed point is a center surrounded by an homoclinic orbit and the shell undergoes non-linear periodic oscillations. However, above this threshold, the fixed point becomes an unstable saddle node, the phase-plane portrait features a non-closed curve and the shell's displacements ultimately diverge.

Haslach and Humphrey [17] studied the transient vibrations of an isotropic incompressible hyperelastic spherical membrane in a fluid under initial perturbation. The membrane is assumed to deform uniformly and keeps a spherical shape for all times. A single non-linear ordinary differential equation driving the dynamics of this membrane is solved. A few material models, such as the Fung and Skalak biological model as well as the neo-Hookean, Mooney-Rivlin, Alexander and Ogden rubber models are considered in order to clarify which one is the most suitable for the description of the dynamic behavior of soft biological tissues. It is shown that rubber structures exhibit bifurcations in their dynamic response that are not present in traditional models for biological tissues.

In works of Zhu et al., the oscillations of a membrane [37] and a balloon [36] made of dielectric elastomer are studied. The non-linearly elastic behavior of the material is derived from an incompressible neo-Hookean strain energy density with an additional term corresponding to the dielectric energy. In [37], the superharmonic, harmonic, and subharmonic resonances, caused by an external sinusoidal pressure are found in agreement with experimental data previously obtained by Fox and Goulbourne [14, 13]. In [36], the stability of the static equilibrium is investigated along with the surrounding oscillations. It is found that the vibration amplitude of the balloon may jump at certain 
values of the excitation frequency, exhibiting hysteresis. However, only a low-dimensional model is used in this study. Complementary results on the dynamics of a dielectric incompressible neo-Hookean spherical shell are reported in [33]: the system is more stable with an increasing thickness of the shell.

Yuan et al. [35] studied the dynamic inflation of infinitely long cylindrical tubes. The inner surface of the tube is subjected to periodic step pressures. The incompressible Ogden material model is implemented and the strain energy density function reduces to neo-Hookean type. Sensitivity of the dynamics to the material parameters, the structure geometry, and the applied pressure is discussed. In [34], the authors explored the dynamic symmetric response of a spherical membrane made of incompressible material with the Rivlin-Saunders constitutive law. The membrane is subjected to periodic step loads on its inner and outer surfaces. A spherically symmetric deformation is assumed and the expressions for principal stretches are derived. The corresponding differential equation that approximately drives the normal oscillation of the spherical membrane is obtained. It is found that periodic oscillations do not exist and that the shell will be fractured ultimately with time when the prescribed external load exceeds a critical threshold that depends on material parameters.

Ogden and Roxburgh studied plane vibrations superimposed to the pure homogeneous deformation of a rectangular plate made of incompressible [24] and compressible [29] hyperelastic materials. The sensitivity of the frequency spectrum to the pre-stress and geometrical parameters for a plane-strain plate problem is provided for neo-Hookean, Varga and Blatz-Ko hyperelastic laws.

Gonçalves et al. [16, 30] investigated linear and non-linear free and forced vibrations of prestretched annular hyperelastic membranes. The membrane material is assumed to be incompressible, homogeneous, isotropic, and is described by a neo-Hookean constitutive law. The in-plane displacements are ignored while the transverse displacement field is approximated by a series of natural modes. Both studies report that a single degree-of-freedom model correctly predicts large vibrations. It is also shown that (i) a lightly stretched membrane displays a highly non-linear hardening response, (ii) the non-linearity decreases as the stretching ratio increases, and (iii) the response becomes essentially linear for a deformed radius of at least twice the initial value. In Ref. [16], a comparison is conducted for different hyperelastic models such as the Mooney-Rivlin, Yeoh, Ogden and Arruda-Boyce models. The results show that the membrane exhibits the same non-linear frequency-amplitude behavior for all tested models, with just a slight difference for the Ogden model.

This literature review highlights the fact that previous works, in a vast majority, employ incompressible neo-Hookean laws to describe rubbers' non-linear elastic behaviors: accordingly, this model is also considered in the present investigation, which extends hyperelastic plate finite amplitude bending vibrations to commonly ignored in-plane displacements. Also, the common and simplifying pre-assumed shape of deformation is discarded here since potential internal resonances can combine several modes in the sought vibratory signatures, making the targeted deformation pattern quite complicated [3]. The present study attempts to overcome the above-mentioned restriction for the large-amplitude static as well as vibratory investigations of a rubber rectangular plate. The von Kármán model is exploited to capture geometrically non-linear bending as it is reputed to be sufficiently accurate for thin isotropic plates [3]. The associated governing equations neglect shear strains and rotary inertia; in addition, thickness stretching/compression cannot be readily recovered and the transverse normal strain has to be determined through additional considerations. Accordingly, the absence of transverse normal stress together with an incompressibility constraint are utilized within a method that iteratively builds a local model capable of accurately approximating the plate's vibratory response around a static deformed configuration. A comparison with a purely geometrically non-linear model is carried out.

\section{Lagrange equations}

The static and dynamic deflections of a thin rubber plate are considered and the well-known Lagrange framework is used to derive the corresponding governing equations of motion:

$$
\frac{\mathrm{d}}{\mathrm{d} t}\left(\frac{\partial L}{\partial \dot{q}_{i}}\right)-\frac{\partial L}{\partial q_{i}}=Q_{i}
$$


where $L=T-\Pi$ is the Lagrange's functional of the system of interest; it comprises the potential energy of elastic deformation $\Pi=\iiint_{V} W \mathrm{~d} v$, integral of the strain energy density $W$ over the volume $V$, and the kinetic energy of the plate, $T$ expressed as [3]:

$$
T=\frac{1}{2} \rho h \iint_{S}\left(\dot{u}^{2}+\dot{v}^{2}+\dot{w}^{2}\right) \mathrm{d} s
$$

where $S$ is the surface area of the middle plane of the plate; $\rho$ is the pass-density per unit length of the plate material, and $h$ is the thickness of the plate. In Eq. (1), the displacements $u(x, y, t)$, $v(x, y, t)$, and $w(x, y, t)$ live in a three-dimensional Euclidian space and are defined in a Cartesian frame $(x, y, t)$ : they will soon be expressed in terms of the generalized coordinates $q_{i}$. The generalized forces are denoted $Q_{i}$.

\section{Geometrically non-linear strain-displacement relationships}

The geometrical non-linearity of the system is reflected through the non-linear von Kármán plate theory [3] yielding the following strain-displacement relationships:

$$
\begin{aligned}
\epsilon_{1} & =\frac{\partial u}{\partial x}+\frac{1}{2}\left(\frac{\partial w}{\partial x}\right)^{2}-z \frac{\partial^{2} w}{\partial x^{2}} \\
\epsilon_{2} & =\frac{\partial v}{\partial y}+\frac{1}{2}\left(\frac{\partial w}{\partial y}\right)^{2}-z \frac{\partial^{2} w}{\partial y^{2}} \\
\epsilon_{12} & =\frac{\partial u}{\partial y}+\frac{\partial v}{\partial x}+\frac{\partial w}{\partial x} \frac{\partial w}{\partial y}-2 z \frac{\partial^{2} w}{\partial x \partial y}
\end{aligned}
$$

where $\epsilon_{1}, \epsilon_{2}$, and $\epsilon_{12}$ are the components of the Lagrange strain tensor for thin plates. The displacements are expanded into truncated series:

$$
\begin{aligned}
& w(x, y, t)=\sum_{i=1}^{N_{W}} q_{i}(t) W_{i}(x, y) \\
& u(x, y, t)=\sum_{i=1}^{N_{U}} q_{i+N_{W}}(t) U_{i}(x, y) \\
& v(x, y, t)=\sum_{i=1}^{N_{V}} q_{i+N_{W}+N_{U}}(t) V_{i}(x, y)
\end{aligned}
$$

where $W_{i}(x, y), U_{i}(x, y)$, and $V_{i}(x, y)$ are admissible space functions that satisfy the homogeneous boundary conditions of the problem. The eigenmodes of the underlying linear system are convenient candidates since they form a complete set. The total number of degrees of freedom is given by $N=N_{W}+N_{U}+N_{V}$.

\section{Physical relations}

\subsection{Strain energy density}

The non-linear elasticity of natural rubbers shall be described by a neo-Hookean constitutive law. In this work, the strain energy density for compressible neo-Hookean material proposed by Bower [7] is preferred:

$$
W=\frac{E}{4(1+v)}\left(\bar{I}_{1}-3\right)+\frac{E}{6(1-2 v)}(J-1)^{2}
$$

where $\bar{I}_{1}=I_{1} J^{-\frac{2}{3}}$, both $I_{1}$ and $J$ respectively being the first invariant and the square root of the third invariant of the right Cauchy-Green deformation tensor $\mathbf{C} ; E$ and $v$ are the Young's modulus 
and Poisson's ratio of plate material, respectively. In case of fully incompressible material (i.e. when $J \rightarrow 1, v \rightarrow 0.5$ ) the second term in (9) is well defined and tends to zero [23, 7].

Results stemming from the neo-Hookean strain energy density (9) and the usual linear strain energy density-i.e. linear elasticity—are compared. For thin plates, the linear strain energy density has the following expression [3]:

$$
W=\frac{E}{4(1+v)}\left(\frac{2}{1-v}\left(\epsilon_{1}^{2}+\epsilon_{2}^{2}+2 v \epsilon_{1} \epsilon_{2}\right)+\epsilon_{12}^{2}\right)
$$

In this context, space and time components of the solution can be uncoupled and the Lagrange equations become simple ordinary differential equations with quadratic and cubic non-linearities [3]:

$$
\ddot{q}_{n}+2 \zeta_{n} \Omega_{n} \dot{q}_{n}+\sum_{i=1}^{N} k_{n i} q_{i}+\sum_{i, j=1}^{N} k_{n i j} q_{i} q_{j}+\sum_{i, j, l=1}^{N} k_{n i j \ell} q_{i} q_{j} q_{\ell}=Q_{n}, \quad n=1, \ldots, N
$$

where $\Omega_{n}$ is the natural frequency of the $n$-th linear mode, and $\zeta_{n}$, the corresponding damping ratio; $k_{n i}, k_{n i j}$, and $k_{n i j l}$ are known coefficients resulting from the integration in space. The generalized forces $Q_{n}$ are calculated by differentiation of the virtual work done by external forces. Governing equations of type (11) are well known and several dedicated techniques are available to find their numerical solution [3].

\subsection{Invariants of the Cauchy-Green deformation tensor}

In order to derive the expressions of the right Cauchy-Green deformation tensor $\mathbf{C}$ invariants in terms of the displacements, the Lagrange strain tensor is introduced in Cartesian coordinates:

$$
\mathbf{E}=\frac{1}{2}\left(\begin{array}{ccc}
2 \epsilon_{1} & \epsilon_{12} & 0 \\
\epsilon_{12} & 2 \epsilon_{2} & 0 \\
0 & 0 & 2 \epsilon_{3}
\end{array}\right)
$$

Expressions of $\epsilon_{1}, \epsilon_{2}$, and $\epsilon_{12}$ (but not $\epsilon_{3}$ ) are listed in equations (3), (4), and (5), respectivly. The right Cauchy-Green deformation tensor $\mathbf{C}$ is then defined as follows [7]:

$$
\mathbf{C}=2 \mathbf{E}+\mathbf{I}=\left(\begin{array}{ccc}
2 \epsilon_{1}+1 & \epsilon_{12} & 0 \\
\epsilon_{12} & 2 \epsilon_{2}+1 & 0 \\
0 & 0 & 2 \epsilon_{3}+1
\end{array}\right)
$$

and its three invariants are easily expressed in terms of the Lagrange strain tensor components:

$$
\begin{aligned}
& I_{1}=\operatorname{tr}(\mathbf{C})=2\left(\epsilon_{1}+\epsilon_{2}+\epsilon_{3}\right)+3 \\
& J^{2}=|\mathbf{C}|=\left(2 \epsilon_{3}+1\right)\left(\left(2 \epsilon_{1}+1\right)\left(2 \epsilon_{2}+1\right)-\epsilon_{12}^{2}\right) \\
& \bar{I}_{1}=I_{1} J^{-\frac{2}{3}}=\frac{2\left(\epsilon_{1}+\epsilon_{2}+\epsilon_{3}\right)+3}{\sqrt[3]{\left(2 \epsilon_{3}+1\right)\left(\left(2 \epsilon_{1}+1\right)\left(2 \epsilon_{2}+1\right)-\epsilon_{12}^{2}\right)}}
\end{aligned}
$$

\subsection{Transverse normal strain}

The expression of the transverse normal strain $\epsilon_{3}$ with respect to the plate's displacements $u, v$, and $w$ has to be determined to later be inserted into Eqs. (14)-(16). In order to derive its expression, two approaches based on distinct assumptions are proposed. The first one uses the hypothesis of zero transverse normal stress, which is a common assumption in thin plates theory. The second approach relies on the incompressibility condition of the plate material. 


\subsubsection{Assumption of zero transverse normal stress}

The condition of zero transverse normal stress is enforced by use of the second Piola-Kirchhoff stress tensor $\mathbf{S}$ whose components are:

$$
S_{i j}=2 \frac{\partial W}{\partial C_{i j}}=\frac{\partial W}{\partial E_{i j}}
$$

where $C_{i j}$ and $E_{i j}$ are the components of tensors $\mathbf{C}$ and $\mathbf{E}$, respectively. The transverse normal strain $\epsilon_{3}$ is deduced from the condition $S_{33}=0$ where $S_{33}$ is expressed as follows:

$$
S_{33}=\frac{\partial W}{\partial E_{33}}=\frac{\partial W}{\partial \epsilon_{3}}=\frac{E}{4(1+v)} \frac{\partial \bar{I}_{1}}{\partial \epsilon_{3}}+\frac{E(J-1)}{3(1-2 v)} \frac{\partial J}{\partial \epsilon_{3}}
$$

The derivatives of the invariants in terms of strain tensor components read:

$$
\begin{aligned}
\frac{\partial \bar{I}_{1}}{\partial \epsilon_{3}} & =\frac{\partial I_{1}}{\partial \epsilon_{3}} J^{-\frac{2}{3}}-\frac{2}{3} I_{1} J^{-\frac{5}{3}} \frac{\partial J}{\partial \epsilon_{3}}=2 J^{-\frac{2}{3}}-\frac{2}{3} I_{1} J^{-\frac{5}{3}} \frac{\left(2 \epsilon_{1}+1\right)\left(2 \epsilon_{2}+1\right)-\epsilon_{12}^{2}}{J} \\
& =2 J^{-\frac{2}{3}}\left(1-\frac{I_{1}\left(\left(2 \epsilon_{1}+1\right)\left(2 \epsilon_{2}+1\right)-\epsilon_{12}^{2}\right)}{3 J^{2}}\right)
\end{aligned}
$$

and:

$$
\frac{\partial J}{\partial \epsilon_{3}}=\frac{2\left(\left(2 \epsilon_{1}+1\right)\left(2 \epsilon_{2}+1\right)-\epsilon_{12}^{2}\right)}{2 \sqrt{\left(2 \epsilon_{3}+1\right)\left(\left(2 \epsilon_{1}+1\right)\left(2 \epsilon_{2}+1\right)-\epsilon_{12}^{2}\right)}}=\frac{\left(2 \epsilon_{1}+1\right)\left(2 \epsilon_{2}+1\right)-\epsilon_{12}^{2}}{J}
$$

Then, Eq. (18) takes the form:

$$
S_{33}=\left(\left(2 \epsilon_{1}+1\right)\left(2 \epsilon_{2}+1\right)-\epsilon_{12}^{2}\right)\left(\frac{E}{3(1-2 \nu)} \frac{J-1}{J}-\frac{E J^{-\frac{2}{3}}}{2(1+\nu)} \frac{I_{1}}{3 J^{2}}\right)+\frac{E J^{-\frac{2}{3}}}{2(1+\nu)}
$$

Since rubber has very low compressibility [7], it seems reasonable to assume that the invariant $J$, which captures the volume change, is close to unity. Accordingly, $J$ is rewritten as $J=1+J^{\prime}$ yielding:

$$
\begin{aligned}
S_{33}= & \frac{E}{2(1+v)}\left(1+J^{\prime}\right)^{-\frac{2}{3}}\left(1-\frac{I_{1}\left(\left(2 \epsilon_{1}+1\right)\left(2 \epsilon_{2}+1\right)-\epsilon_{12}^{2}\right)}{3\left(1+J^{\prime}\right)^{2}}\right) \\
& +\frac{E}{3(1-2 v)} \frac{J^{\prime}\left(\left(2 \epsilon_{1}+1\right)\left(2 \epsilon_{2}+1\right)-\epsilon_{12}^{2}\right)}{\left(1+J^{\prime}\right)}
\end{aligned}
$$

where $J^{\prime}$ is assumed to be small. This allows for an expansion of (22) as a truncated series in $J^{\prime}$ :

$$
\begin{aligned}
S_{33}= & \frac{E}{2(1+\nu)}\left(1-\frac{2}{3} J^{\prime}+\frac{5}{9} J^{\prime 2}+\frac{40}{81} J^{\prime 3}-\frac{110}{243} J^{\prime 4}+\ldots\right) \times \\
& \left(1-\frac{I_{1}}{3}\left(\left(2 \epsilon_{1}+1\right)\left(2 \epsilon_{2}+1\right)-\epsilon_{12}^{2}\right)\left(1-2 J^{\prime}+3 J^{\prime 2}-4 J^{\prime 3}+5 J^{\prime 4}+\ldots\right)\right) \\
& +\frac{E}{3(1-2 \nu)}\left(\left(2 \epsilon_{1}+1\right)\left(2 \epsilon_{2}+1\right)-\epsilon_{12}^{2}\right)\left(J^{\prime}-J^{\prime 2}+J^{\prime 3}-J^{\prime 4}+\ldots\right)
\end{aligned}
$$

Invariant $J^{2}$ is recast in the following form

$$
\begin{aligned}
J^{2} & =\left(2 \epsilon_{3}+1\right)\left(\left(2 \epsilon_{1}+1\right)\left(2 \epsilon_{2}+1\right)-\epsilon_{12}^{2}\right) \\
& =1+2\left(\epsilon_{1}+\epsilon_{2}+\epsilon_{3}\right)+4\left(\epsilon_{1} \epsilon_{2}+\epsilon_{1} \epsilon_{3}+\epsilon_{2} \epsilon_{3}\right)-\epsilon_{12}^{2}+8 \epsilon_{1} \epsilon_{2} \epsilon_{3}-2 \epsilon_{12}^{2} \epsilon_{3} \\
& =1+J^{A}
\end{aligned}
$$


We can subsequently expand $J$ around unity:

$$
J=\sqrt{1+J^{A}} \approx 1+\frac{J^{A}}{2}-\frac{\left(J^{A}\right)^{2}}{8}+\frac{\left(J^{A}\right)^{3}}{16}-\frac{5\left(J^{A}\right)^{4}}{128}+\ldots
$$

and then approximate $J^{\prime}$ as follows:

$$
J^{\prime} \approx \frac{J^{A}}{2}-\frac{\left(J^{A}\right)^{2}}{8}+\frac{\left(J^{A}\right)^{3}}{16}-\frac{5\left(J^{A}\right)^{4}}{128}+\ldots
$$

The transverse normal strain $\epsilon_{3}$ is finally represented as a polynomial in the strain components $\epsilon_{1}, \epsilon_{2}$, and $\epsilon_{12}$ :

$$
\epsilon_{3}=\sum_{i \in\{1,2,12\}} \lambda_{i} \epsilon_{i}+\sum_{i, j \in\{1,2,12\}} \lambda_{i, j} \epsilon_{i} \epsilon_{j}+\sum_{i, j, k \in\{1,2,12\}} \lambda_{i, j, k} \epsilon_{i} \epsilon_{j} \epsilon_{k}+\sum_{i, j, k, m \in\{1,2,12\}} \lambda_{i, j, k, m} \epsilon_{i} \epsilon_{j} \epsilon_{k} \epsilon_{m}+\ldots
$$

In (27), there is no constant term because $\epsilon_{3}=0$ when $\epsilon_{1}=\epsilon_{2}=\epsilon_{12}=0$.

Expressions for $J^{A}, J^{\prime}$, and $\epsilon_{3}$ given in (25), (26), and (27) are inserted into (23) and the coefficients associated to the strain terms of the same power are balanced leading to a system of linear algebraic equations in the unknown $\lambda$-coefficients defined in Eq. (27). These systems and expressions for the coefficients are detailed in Appendix A.

\subsubsection{Assumption of incompressibility}

The incompressibility condition yields $J=1$ [23]. By assuming the absence of hydrostatic pressure, the expression of $\epsilon_{3}$ can readily be deduced from Eq. (15):

$$
\epsilon_{3}=\frac{1}{2\left(\left(2 \epsilon_{1}+1\right)\left(2 \epsilon_{2}+1\right)-\epsilon_{12}^{2}\right)}-\frac{1}{2}
$$

Since the Poisson ratio is $v=0.5$ for an incompressible material, the corresponding neo-Hookean strain energy density (9) for thin plates becomes:

$$
W=\frac{E}{3}\left(\epsilon_{1}+\epsilon_{2}+\frac{1}{2\left(\left(2 \epsilon_{1}+1\right)\left(2 \epsilon_{2}+1\right)-\epsilon_{12}^{2}\right)}-\frac{1}{2}\right)
$$

The series expansion of (29) up to the second powers in the strain components coincides with expression (10) (see Appendix B for details). That is, for small strains, both theories give identical equations which also means that these theories are consistent.

The expansion of (28) in a series around zero has the form:

$$
\begin{aligned}
\epsilon_{3} \approx & -\left(\epsilon_{1}+\epsilon_{2}\right)+2\left(\epsilon_{1}^{2}+\epsilon_{2}^{2}+\epsilon_{1} \epsilon_{2}\right)+\frac{\epsilon_{12}^{2}}{2}-4\left(\epsilon_{1}^{3}+\epsilon_{2}^{3}+\epsilon_{1}^{2} \epsilon_{2}+\epsilon_{1} \epsilon_{2}^{2}\right)-2\left(\epsilon_{1} \epsilon_{12}^{2}+\epsilon_{2} \epsilon_{12}^{2}\right) \\
& +8\left(\epsilon_{1}^{4}+\epsilon_{2}^{4}+\epsilon_{1}^{3} \epsilon_{2}+\epsilon_{1} \epsilon_{2}^{3}+\epsilon_{1} \epsilon_{2} \epsilon_{12}^{2}\right)+6\left(\epsilon_{1}^{2} \epsilon_{12}^{2}+\epsilon_{2}^{2} \epsilon_{12}^{2}\right)+\frac{\epsilon_{12}^{4}}{2}
\end{aligned}
$$

Surprisingly, the coefficients in (30) coincide with the coefficients of expansion (27) when it is assumed $v=0.5$. Accordingly, a quite interesting feature is observed: both approaches lead to the same expansion for incompressible materials.

\section{Local expansion of the neo-Hookean strain energy density}

Expression (29) is not a polynomial in strains, which essentially complicates the investigation of the plate's behavior. Solutions of such non-linear systems are conceivable only numerically, and still for low-dimensional models. As a first approach, substitution of the truncated series (30) into (29) allows one to obtain $W$ in a polynomial form and, hence, the Lagrange equations in this case are ordinary 
differential equations with higher-order (higher than three) polynomial non-linearities. However, higher-order equations are not desirable since their integration quickly becomes tedious as their size blows up with the number of degrees of freedom $N$.

In order to simplify the analysis, expression (29) is first transformed. In particular, governing equations in the form of ordinary differential equations with polynomial non-linearities of order not higher than three are targeted. It is worth noting that such a model will capture the dynamic response of the plate in the vicinity of a certain static configuration only. A certain static deformed configuration is supposed to be known and identified by a set of generalized coordinates $\mathbf{q}^{(0)}=\left\{q_{i}{ }^{(0)}\right\}_{i=1, \ldots, N}$ around which a new deformed configuration $\mathbf{q}=\left\{q_{i}\right\}_{i=1, \ldots, N}$ is to be calculated:

$$
\mathbf{q}=\mathbf{q}^{(0)}+\alpha \mathbf{q}^{(1)}
$$

where $\alpha \ll 1$ is a small formal parameter. The strain components can be expressed as:

$$
\epsilon_{1}=\epsilon_{1}^{(0)}+\alpha \epsilon_{1}^{(1)} \quad ; \quad \epsilon_{2}=\epsilon_{2}^{(0)}+\alpha \epsilon_{2}^{(1)} \quad ; \quad \epsilon_{12}=\epsilon_{12}^{(0)}+\alpha \epsilon_{12}^{(1)}
$$

In expressions (32), all $\alpha$-dependent terms are included in $\alpha \epsilon_{i}^{(1)}$ and the quantities $\epsilon_{1}^{(0)}, \epsilon_{2}^{(0)}, \epsilon_{12}^{(0)}$ do not depend on the unknown generalized coordinates $\mathbf{q}^{(1)}$.

Expression (29) is then expanded as a series in the small parameter $\alpha$, keeping terms up to the second power:

$$
W\left(\mathbf{q}^{(0)}\right)=\frac{E}{3}\left(\left[\epsilon_{1}^{(0)}+\epsilon_{2}^{(0)}+\frac{1}{2 \Xi}-\frac{1}{2}\right]+\alpha\left(\epsilon_{1}^{(1)}+\epsilon_{2}^{(1)}+\frac{\kappa}{\Xi^{2}}\right)+\alpha^{2}\left(\frac{4 \kappa^{2}-\left(4 \epsilon_{1}^{(1)} \epsilon_{2}^{(1)}-\left(\epsilon_{12}^{(1)}\right)^{2}\right) \Xi}{2 \Xi^{3}}\right)\right)
$$

where:

$$
\Xi=\left(2 \epsilon_{1}^{(0)}+1\right)\left(2 \epsilon_{2}^{(0)}+1\right)-\left(\epsilon_{12}^{(0)}\right)^{2} ; \kappa=\epsilon_{1}^{(1)}+\epsilon_{2}^{(1)}+2 \epsilon_{1}^{(0)} \epsilon_{2}^{(1)}+2 \epsilon_{2}^{(0)} \epsilon_{1}^{(1)}-\epsilon_{12}^{(0)} \epsilon_{12}^{(1)}
$$

The small parameter $\alpha$ is only formal. In the sequel, the additional deflection $\mathbf{q}^{(1)}$ is assumed to be small with respect to $\mathbf{q}^{(0)}$.

The expression in square brackets in (33) does not depend on the generalized coordinates and vanish during differentiation. The denominators in (33) do not depend on the unknown vector of generalized coordinates $\mathbf{q}^{(1)}$ and Eq. (33) is thus polynomial in $q_{i}{ }^{(1)}$. Once the space and time participations are separated, Eqs. (11) take the following form:

$$
\ddot{q}_{n}^{(1)}+2 \zeta_{n} \Omega_{n} \dot{q}_{n}^{(1)}+\sum_{i=1}^{N} k_{n i}\left(\mathbf{q}^{(0)}\right) q_{i}^{(1)}+\sum_{i, j=1}^{N} k_{n i j}\left(\mathbf{q}^{(0)}\right) q_{i}^{(1)} q_{j}^{(1)}+\sum_{i, j, \ell=1}^{N} k_{n i j \ell}\left(\mathbf{q}^{(0)}\right) q_{i}^{(1)} q_{j}^{(1)} q_{\ell}^{(1)}=Q_{n}
$$

for $n=1, \ldots, N$. These equations stand for the so-called local model which describes the behavior of the plate around the deformed static configuration $\mathbf{q}^{(0)}$. The latter is calculated through a sequence of steps that are now detailed. The external static load is indicated by a single-value parameter $P$ and a deformed configuration $\mathbf{q}^{(0)}$ is known by assumption:

Step 1. The static counterpart of (35) where $\ddot{\mathbf{q}}^{(1)}=\dot{\mathbf{q}}^{(1)}=\mathbf{0}$ is built according to the strain energy density (33). A total of $N+1$ unknowns ( $N$ generalized coordinates stored in $\mathbf{q}^{(1)}$ together with the applied load $P$ ) are identified.

Step 2. By choice, the first generalized coordinate is enforced to be $q_{1}{ }^{(1)}=H$ with $H \ll q_{1}{ }^{(0)}$. The number of equations balances the number of unknowns. 
Step 3. The system of equations is solved using the Newton-Raphson method and generalized coordinates $q_{i}{ }^{(1)}, i=2, \ldots, N$ as well as the applied force $P$ are determined.

Step 4. The static configuration is updated through $\mathbf{q}^{(0)} \leftarrow \mathbf{q}^{(0)}+\mathbf{q}^{(1)}$ before going back to Step 1 of the next iteration.

The iterations continue until a desired deflection is reached. Overall, the above strategy is a sequential Newton-Raphson procedure in which, one of the initial unknowns, namely $q_{1}^{(1)}$ is iteratively increased and advantageously replaced by the applied external pressure $P$ in the list of unknowns.

\section{Exact low-dimensional models with both material and geometric non-linearities}

Here we describe an approach that allows one to check the relevance and accuracy of the approximate numerical solution obtained with the local models method previously detailed. This approach consists of the numerical solution to the Lagrange Eqs. (1) in the static case with strain energy density (29). Since the latter is a smooth function in the generalized coordinates, integration and differentiation commute:

$$
\frac{\partial}{\partial \mathbf{q}}\left(\iiint_{V} W \mathrm{~d} v\right)=\iiint_{V} \frac{\partial W}{\partial \mathbf{q}} \mathrm{d} v=\mathbf{Q}
$$

For a prescribed pressure $P$, the current numerical solution $\mathbf{q}$ is substituted into (36). Accordingly, $\frac{\partial W}{\partial q_{i}}$ becomes a function of the space coordinates only and it is possible to perform the full threedimensional numerical integration of Eqs. (36). The resulting system of algebraic equations is solved through the Newton-Raphson method and the corresponding solution is named the exact solution hereinafter. However, system (36) is computationally expensive and it is possible to solve it only for few degrees of freedom.

\section{Numerical example}

\subsection{Static analysis}

As an example, a simply supported rectangular rubber plate illustrated in Fig. 1 is considered. It is defined on the following domain:

$$
V=\{x \in[0 ; a], y \in[0 ; b], z \in[-h / 2 ; h / 2]\}
$$

with the geometrical parameters $a=0.1 \mathrm{~m}, b=0.12 \mathrm{~m}, h=5 \cdot 10^{-3} \mathrm{~m}$, and material characteristics $v=0.5, E=10^{7} \mathrm{~Pa}, \rho=1100 \mathrm{~kg} / \mathrm{m}^{3}$. The plate is simply supported with immovable edges thus

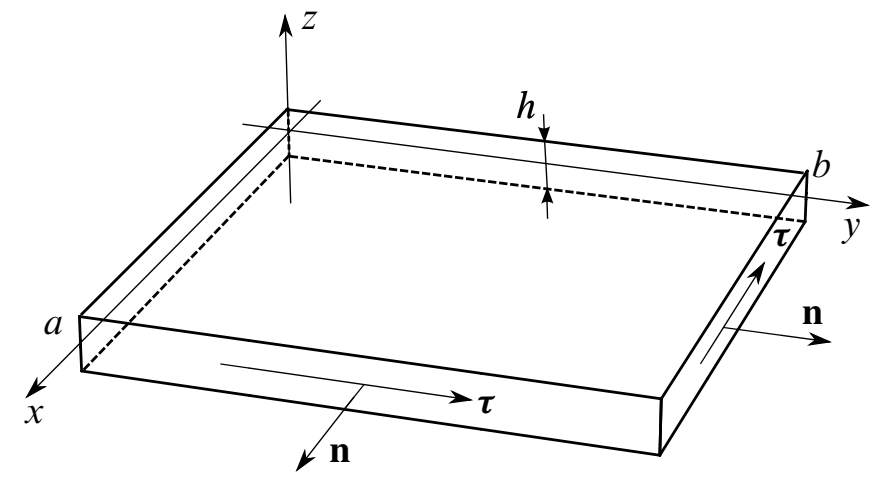

Figure 1. Plate of interest and corresponding coordinate system

producing the common boundary conditions [3]:

$$
\left.w\right|_{\partial S}=\left.M\right|_{\partial S}=\left.u\right|_{\partial S}=\left.v\right|_{\partial S}=0
$$




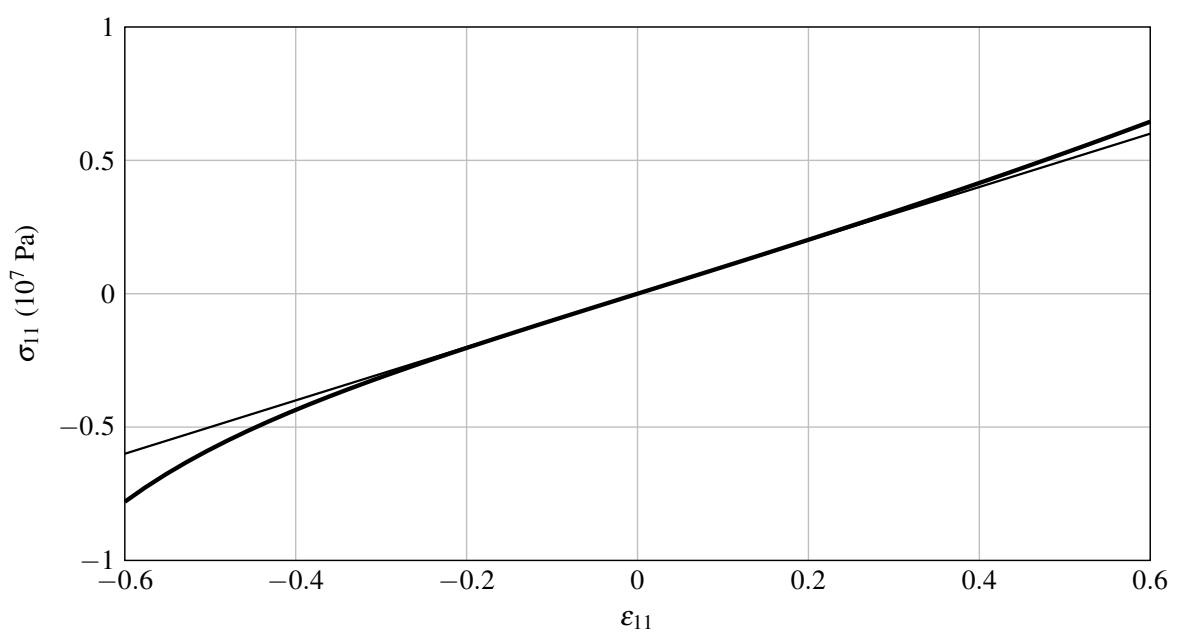

Figure 2. Cauchy stress-strain diagram for uniaxial tension: — neo-Hookean law; — Hooke's law

where $\partial S$ denotes the boundary of the plate. The associated stress-strain diagram for uniaxial tension and compression is depicted in Fig. 2. The bending moment per unit length $M$ reads [3]:

$$
M=-D\left(\frac{\partial^{2} w}{\partial n^{2}}+v \frac{\partial^{2} w}{\partial \tau^{2}}\right)
$$

where $\mathbf{n}$ and $\boldsymbol{\tau}$ are the outer normal and tangent directions to $\partial S$, respectively. The corresponding linear eigenmodes are simple sine functions [3]:

$$
\begin{aligned}
& w(x, y, t)=\sum_{n, m \in \mathbb{N}} w_{n, m}(t) \sin \left(\frac{n \pi x}{a}\right) \sin \left(\frac{m \pi y}{b}\right) \\
& u(x, y, t)=\sum_{n, m \in \mathbb{N}} u_{n, m}(t) \sin \left(\frac{n \pi x}{a}\right) \sin \left(\frac{m \pi y}{b}\right) \\
& v(x, y, t)=\sum_{n, m \in \mathbb{N}} v_{n, m}(t) \sin \left(\frac{n \pi x}{a}\right) \sin \left(\frac{m \pi y}{b}\right)
\end{aligned}
$$

The deflection under uniformly distributed constant pressure $P$ is now investigated. Due to symmetry considerations on the geometry of the plate as well as on the distribution of the external load, the odd bending modes are solely participating into the solution when no internal resonances are activated:

$$
w(x, y, t)=\sum_{n, m \in \mathbb{N}} w_{2 n+1,2 m+1}(t) \sin \left(\frac{(2 n+1) \pi x}{a}\right) \sin \left(\frac{(2 m+1) \pi y}{b}\right)
$$

and respective in-plane modes in the solution have the form $[3,9]$ :

$$
\begin{aligned}
& u(x, y, t)=\sum_{n, m \in \mathbb{N}} u_{2 n, 2 m+1}(t) \sin \left(\frac{2 n \pi x}{a}\right) \sin \left(\frac{(2 m+1) \pi y}{b}\right) \\
& v(x, y, t)=\sum_{n, m \in \mathbb{N}} v_{2 n+1,2 m}(t) \sin \left(\frac{(2 n+1) \pi x}{a}\right) \sin \left(\frac{2 m \pi y}{b}\right)
\end{aligned}
$$

The problem is also appropriately scaled as detailed below:

$$
\begin{aligned}
q_{i}=\frac{w_{2 n+1,2 m+1}}{h}, & i=1, \ldots, N_{W} \\
q_{i}=\frac{u_{2 n, 2 m+1}}{h}, & i=N_{W}+1, \ldots, N_{W}+N_{U} \\
q_{i}=\frac{v_{2 n+1,2 m}}{h}, & i=N_{W}+N_{U}+1, \ldots, N
\end{aligned}
$$


and $\tau=\Omega_{1} t$ where $\Omega_{1}$ is the circular frequency of the first natural mode of the deformed plate. In expressions (45) the two-index generalized coordinates $w_{2 n+1,2 m+1}, u_{2 n, 2 m+1}, v_{2 n+1,2 m}$ are replaced by single-index coordinates $q_{i}$. For each particular aspect ratio and thickness of the plate, different terms have to be considered in expansions (43) and (44) in order to reach a good accuracy.

\subsubsection{Geometrically non-linear bending}

Attention is paid to strain energy density (10) where geometrical non-linearities only are considered. The results are obtained for an increasing number of generalized coordinates in (43) and (44). In Fig. 3

\begin{tabular}{ll}
\hline Size & Participating eigenmodes \\
\hline 3 dofs & $w_{1,1}, u_{2,1}, v_{1,2}$ \\
12 dofs & $w_{i, j}, i, j=1,3 ; u_{i, j}, v_{j, i}, i=2,4 ; j=1,3$ \\
27 dofs & $w_{i, j}, i, j=1,3, u_{i, j}, v_{j, i}, i=2,4,6 ; j=1,3,5$ \\
34 dofs & $w_{i, j}, i, j=1,3,5,7 ; u_{i, j}, v_{j, i}, i=2,4,6 ; j=1,3,5$ \\
\hline
\end{tabular}

Table 1. Eigenmodes used for models with increasing numbers of dofs

the central deflection of the plate with respect to the applied pressure is depicted. For each curve, the participating eigenmodes are listed in Table 1.

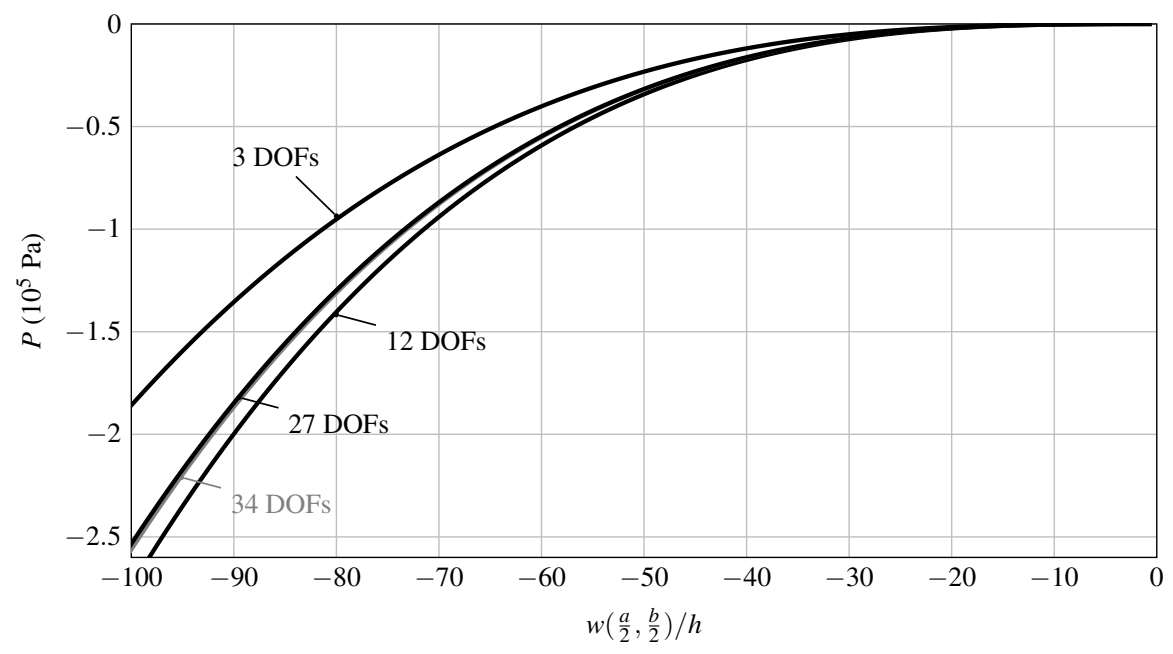

Figure 3. Deflection-pressure curves for models with geometrical non-linearity only

The convergence is such that the various approximations approach the solution from alternating sides. The 27-dof solution lies between the 3-dof and 12-dof solutions, but closer to the 12-dof solution; similarly, the 34-dof solution lies between the 12-dof and 27-dof solutions, but closer to the 27-dof solution. The maximal difference between the 12-dof and 34-dof models for a given pressure $P$ in the range of deflections $[0,100 \mathrm{~h}]$ is $2.2 \%$. Accordingly, it is understood that the 12 -dof is sufficiently accurate and solely investigated in the sequel.

\subsubsection{Physically and geometrically non-linear bending}

Exact model The results for systems with 3,12, 27 and 34 dofs with both types of non-linearities are obtained by the local model method (see section 4) and the strain energy density (9) is used. Also, the exact solutions (see section 5) are found for the 3 and 12 dof systems. The participating modes in this subsection and in the remainder of the paper are the ones listed in Table 1.

The comparison of deflection-pressure curves for the model with only geometrical non-linearity and exact solution for the 12 dof systems is shown in Fig. 4. We can see that the difference within a range of deflection up to $30 \mathrm{~h}$ is small. Only a $6 \%$ maximal difference for a given pressure is observed in this range. 


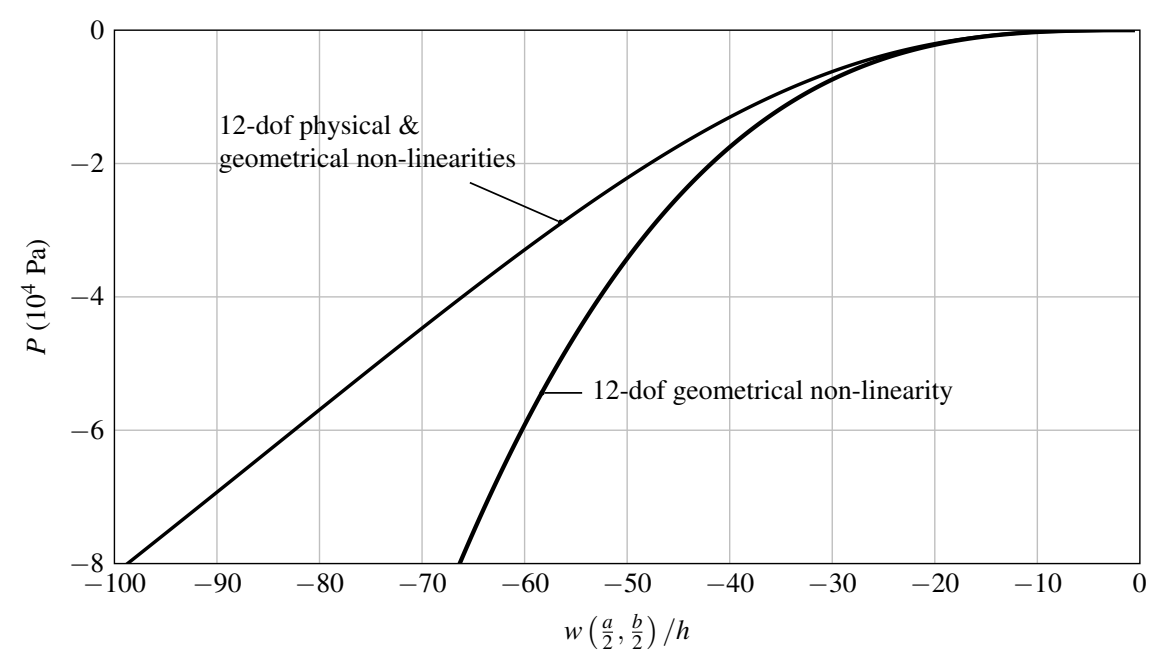

Figure 4. Comparison of the exact deflection-pressure curves considering only geometrical and both geometrical and physical non-linearities; 12 dofs

The strain intensity over the thin plate is defined as [19]:

$$
\epsilon_{\mathrm{INT}}=\sqrt{\frac{2}{3}} \sqrt{\left(\epsilon_{1}-\epsilon_{2}\right)^{2}+\left(\epsilon_{1}-\epsilon_{3}\right)^{2}+\left(\epsilon_{2}-\epsilon_{3}\right)^{2}+3 / 2 \epsilon_{12}^{2}}
$$

For a central deflection of $30 h, \max _{x, y, z} \epsilon_{\mathrm{INT}}=0.1$. At higher strains, the effect of physical nonlinearity is significant. This result was expected due to the asymptotic expansion of the neo-Hookean strain energy density, as discussed in the Appendix B. The powers up to second order in the asymptotic expansion for the neo-Hookean strain energy density coincide with the physically linear strain energy density. These powers properly reflect the behavior at small strains. However, the neo-Hookean strain energy density expansion contains higher powers, which causes the difference at moderate strains.

Local model As displayed in Fig. 4, for deflections up to 30h, the model with only geometrical nonlinearity satisfactorily approximates the solution involving both non-linearities. Therefore numerical iterations to obtain deflection curves are started at a deformation of $30 \mathrm{~h}$ in Fig. 5 and only exact solutions are shown for smaller deflection.

Fig. 5 compares the corresponding results with both non-linearities and an increasing number of dofs to the available exact solutions. It is shown that the local model provides a sufficiently good approximation of the underlying plate behavior. Again, the 12 dof model stands as a convincing compromise between prediction capabilities and computational cost. The maximal difference in deflection in the range of deflection $[0,100 h]$ is $2 \%$ with respect to the 34 dof model.

\subsection{Dynamic analysis}

We study the free and forced vibrations around a pre-loaded state, where physical non-linearity has significant effect. The initial deformed configuration is such that $w_{1,1}=80 \mathrm{~h}$ as illustrated in Fig. 6. Comparison with the exact static solution shows that the local model is accurate for deflections up to $10 h$, limit of our dynamic analysis.

\subsubsection{Free and forced vibrations}

The harmonic balance method [25] is applied to obtain periodic solutions of the system of type (35) through a Fourier expansion of the generalized coordinates:

$$
q_{i}(\tau)=A_{i, 0}+\sum_{j=1}^{N_{h}} A_{i, j} \cos (j \Omega \tau), \quad i=1, \ldots, 12
$$




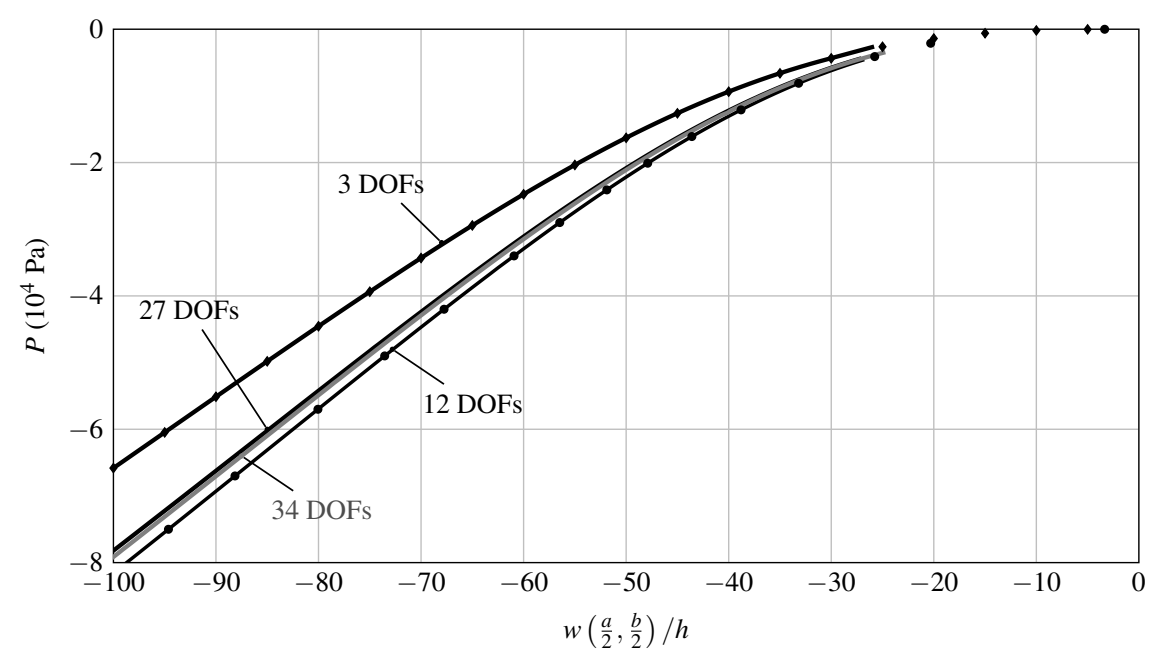

Figure 5. Deflection-pressure curves with physical and geometrical non-linearities. Local models:,-- and exact solution: $\bullet$ dofs, $\bullet 12$ dofs

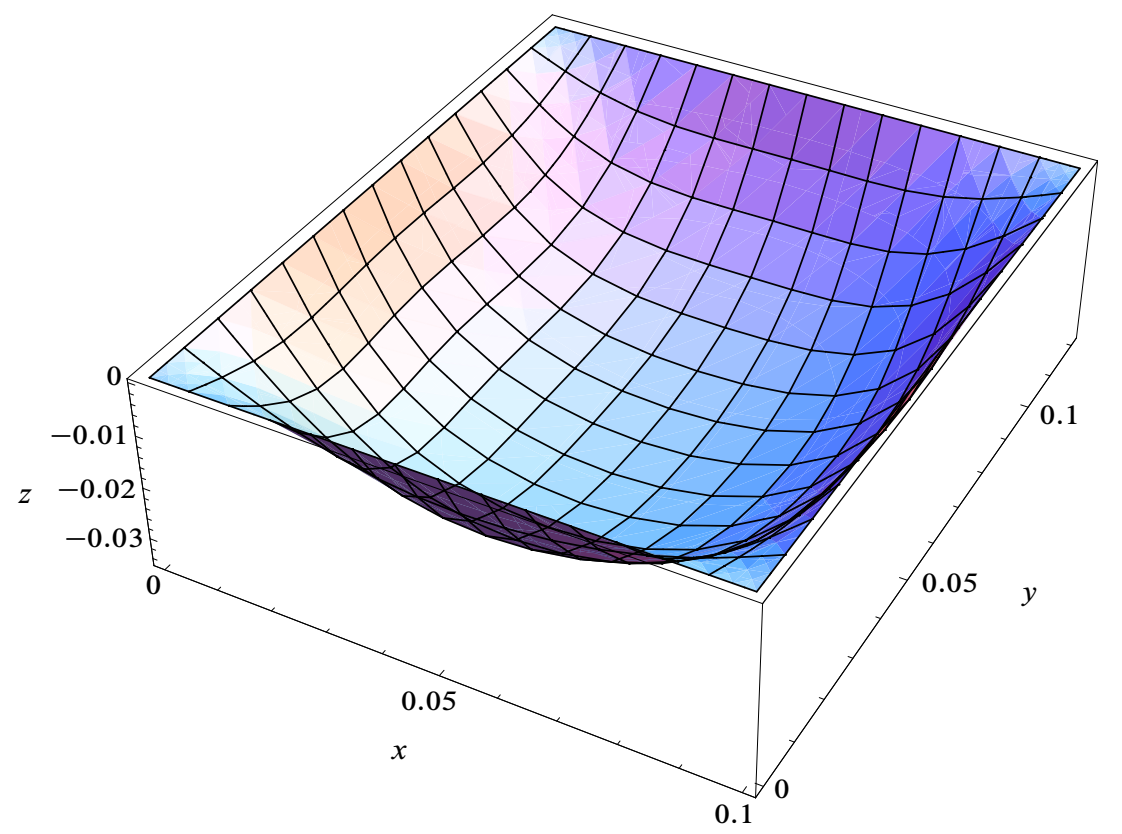

Figure 6. Deflected plate: the deflection is of the order of the plate in-plane size

where $\Omega$ is the non-dimensional frequency, normalized with respect to $\Omega_{1}$. Harmonics $A_{i, j}$ are determined from the system of non-linear algebraic equations written by balancing of coefficients associated to the same harmonics in equations (11) after substitution into Eq. (47). A convergence analysis shows that $N_{h}=8$ in (47) is a good compromise.

Fig. 7 displays the forced response to a periodic pressure excitation of amplitude $P_{d}=26.5 \mathrm{~Pa}$ with damping ratio $\zeta_{n}=\zeta=0.001$, as well as the corresponding backbone curves of the free response around the first eigenfrequency of the pre-loaded plate illustrated in Fig. 6. The response exhibits two peaks, the first one being associated to the 2:1 internal resonance with in-plane mode $v_{1,2}$ (softening branch in Fig. 7), and two Neimark-Sacker bifurcations at $\Omega=0.9981$ and $\Omega=1$. Stable and unstable orbits are shown by solid and dashed lines, respectively. The stability analysis is based on the calculation of frequency multipliers [25, 32]. The forced response between the two Neimark-Sacker bifurcations is quasi-periodic, i.e. it features amplitude modulations.

The analysis of the linearized version of system (35) shows that the first eigenfrequency of the deformed plate is equal to $2302.12 \mathrm{rad} / \mathrm{s}$, about 86 times greater than first eigenfrequency of the flat 


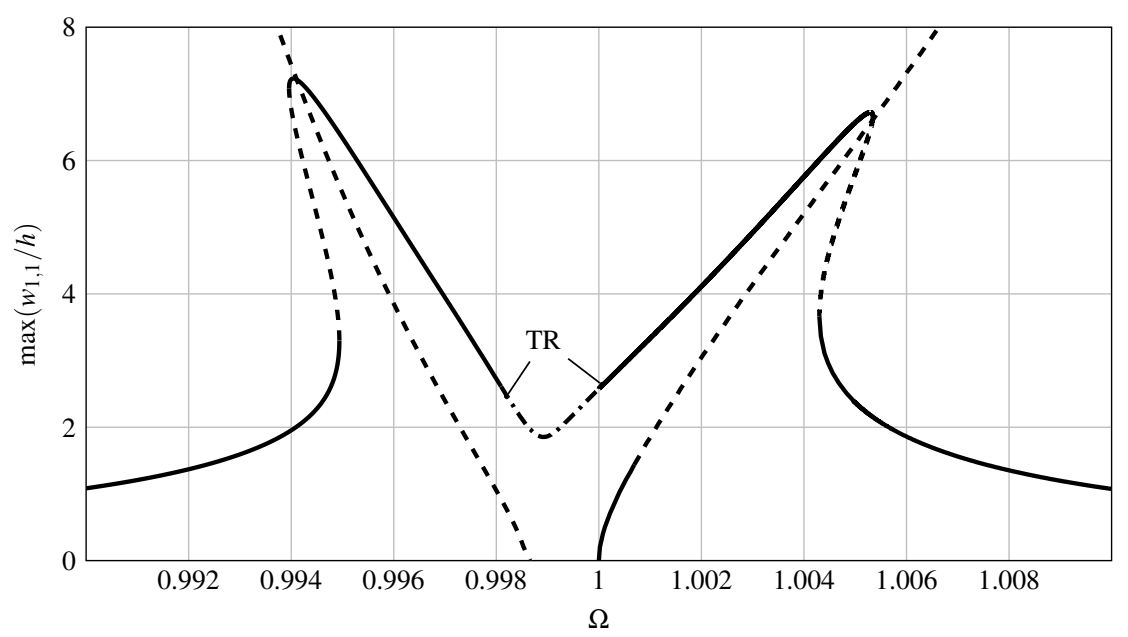

Figure 7. Forced response and backbone curves for principal bending mode $w_{1,1}$ : — stable motions; - - unstable motions; - - - quasi-periodic motions; TR, Neimark-Sacker bifurcation. $P_{d}=26.5 \mathrm{~Pa}, \zeta=0.001$

plate given by [22]:

$$
\Omega_{1 \text { flat }}=h \pi^{2} \sqrt{\frac{E}{12 \rho\left(1-v^{2}\right)}}\left(\frac{1}{a^{2}}+\frac{1}{b^{2}}\right)
$$

It is well-known that the amplitude-frequency relationship for simply supported flat plates is highly non-linear [5, 4]. However, the deformed plate under analysis exhibits very weak non-linearity (the difference between frequency of vibrations with amplitude $10 \mathrm{~h}$ and linear eigenfrequency is only $0.8 \%$ ), but the behavior of the plate is not that of simple linear oscillations. As already mentioned, interaction with in-plane modes is observed in both backbone curves, shown in Fig. 7. This effect of weaken non-linearity in stretched membranes is reported in [16, 30].

In order to verify the harmonic balance solution, the exact system of equations:

$$
\left.\frac{\mathrm{d}}{\mathrm{d} t}\left(\frac{\partial T}{\partial \dot{\mathbf{q}}}\right)\right|_{\mathbf{q}_{0}}+\left.\iiint_{V} \frac{\partial W}{\partial \mathbf{q}}\right|_{\mathbf{q}_{0}} \mathrm{~d} v=\mathbf{0}
$$

is numerically integrated through the Runge-Kutta time-marching technique for free vibrational response. Prescribed initial displacements are obtained from the truncated Fourier series (47) at $\tau=0$. Initial velocities are zero. The central deflection versus time is displayed in Fig. 8(a). This figure shows good agreement between the time response of the exact model and the periodic response predicted by the combination of the local model and harmonic balance. It should be mentioned that the vibrations are non-symmetric with respect to the mean value of the response. Fig. 8(b) shows the contribution of main harmonics to the vibration response at the center of the plate. There appears to be a significant contribution of second harmonic in the response, explaining the asymmetry of the vibrations which stemms from the initially curved shape of the plate initiated by the external static pressure.

The forced vibrations are further explored through the AUTO software [12]. External forcing comes from a time-dependent periodic pressure with a constant mean-value that corresponds to the deformed configuration around which the local model was built. The frequency responses for principal bending and in-plane generalized coordinates are shown in Figures 9; Fig. 9(b) shows the minimum of $w_{1,1} / h$. From Fig. 9(a) with Fig. 9(b), is noticed again the asymmetric deflection of the plate with respect to the static configuration. Fig. 7 shows the magnification of the part of frequency response from Fig. 9(a). As can be seen from Fig. 7, the backbone curves and forced response agree well. Strong coupling between bending and in-plane coordinates is observed for both branches near the first eigenfrequency. Concerning participations $u_{2,1}$ and $v_{1,2}$, the softening branch is predominant in the 


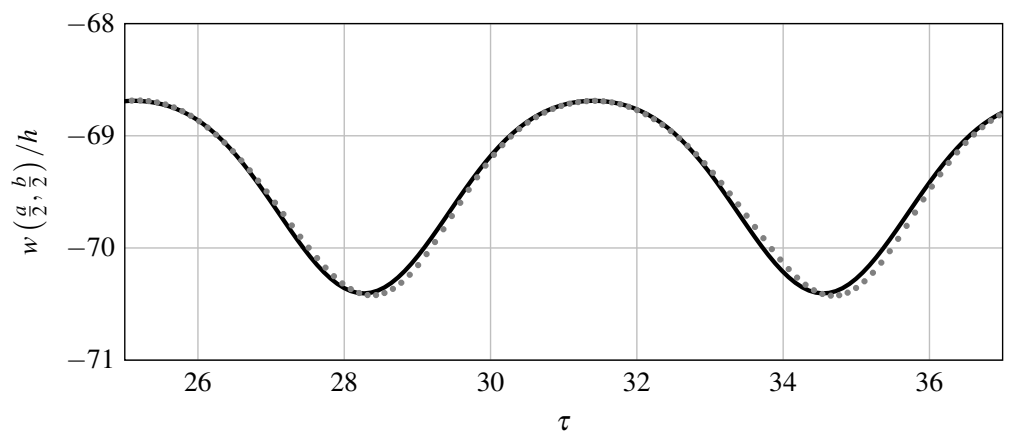

(a) response versus non-dimensional time, — harmonic balance solution, • numerical solution of the exact system

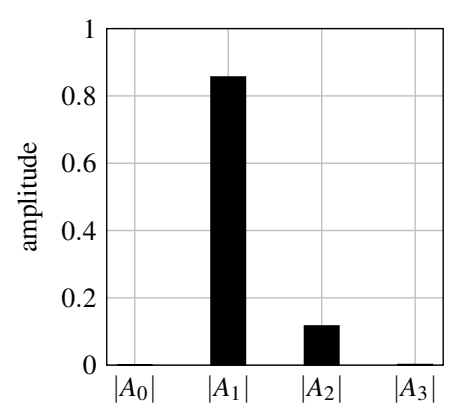

(b) main harmonics

Figure 8. Deflection at the center of the plate

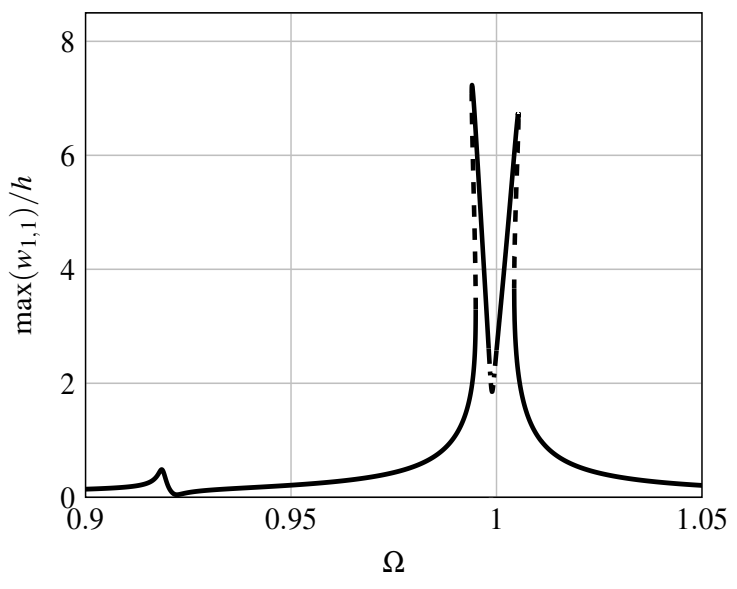

(a) Maximum of bending mode $w_{1,1}$

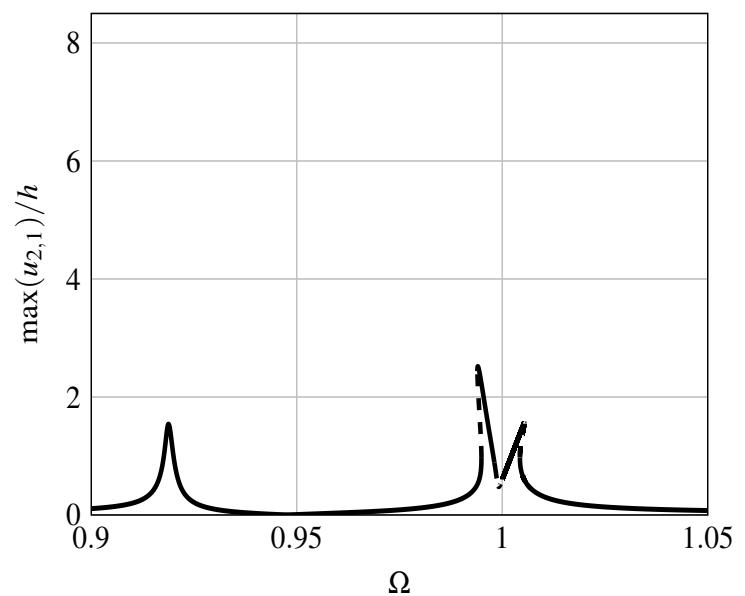

(c) Maximum of in-plane mode $u_{2,1}$

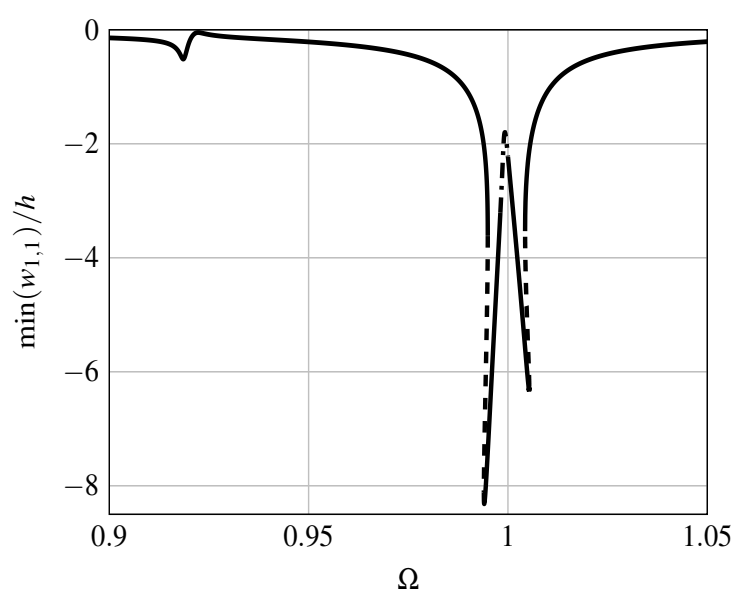

(b) Minimum of bending mode $w_{1,1}$

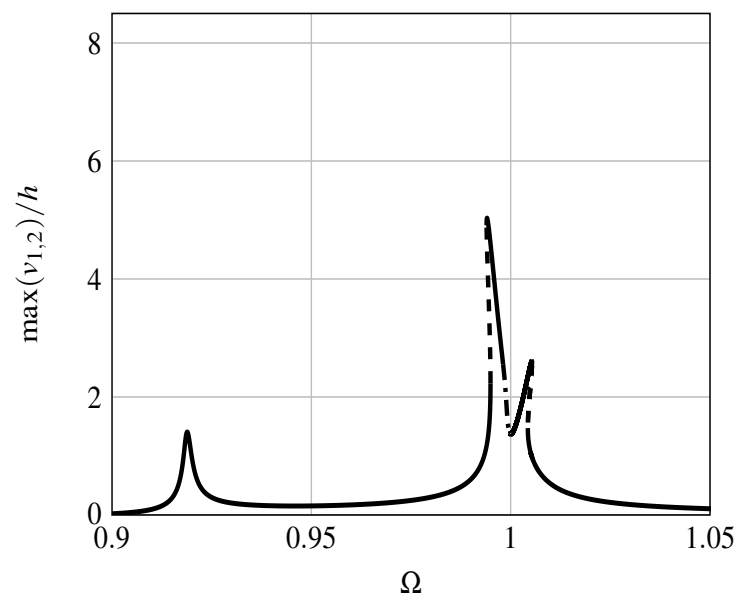

(d) Maximum of in-plane mode $v_{1,2}$

Figure 9. Frequency responses: — stable motions; - - - unstable motions; - - q quasi-periodic motions. $P_{d}=26.5 \mathrm{~Pa}, \zeta=0.001$

forced response because the frequency of vibration in this regime is close to a half of the first in-plane eigenfrequency. 


\subsubsection{A posteriori reduced-order models}

A posteriori inspection of the contribution of the generalized coordinates in the free response of the plate is carried out. Fig. 10 shows four generalized coordinates with respect to the principal bending coordinate $w_{1,1}$ and its derivative with respect to time $\dot{w}_{1,1}$ along the hardening-type regime depicted in Fig. 7. The two coordinates $w_{1,3}$ and $v_{1,2}$ are non-linear with respect to $w_{1,1}$ and $\dot{w}_{1,1}$, while $u_{2,1}$ and $u_{4,1}$ are linear, as illustrated in Figures 10(b) and 10(c). The dependences of the remaining generalized coordinates on $w_{1,1}$ and $\dot{w}_{1,1}$ are also almost linear ones and the amplitudes of these coordinates are much smaller compared to the amplitudes of the major contributions $w_{1,1}, w_{1,3}, u_{2,1}$ and $v_{1,2}$. This paves the way to further reduction of the model dimension.

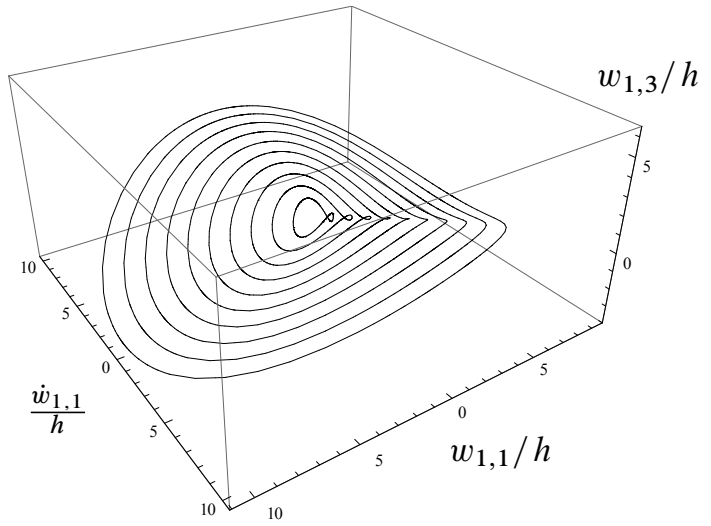

(a) $w_{1,3}$

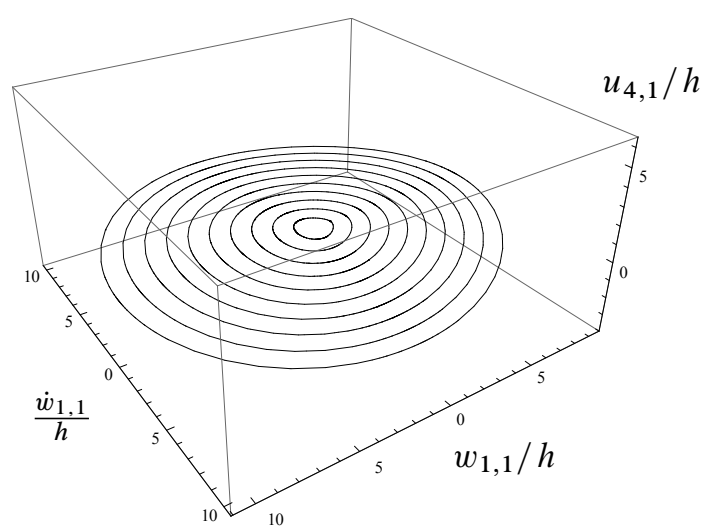

(c) $u_{4,1}$

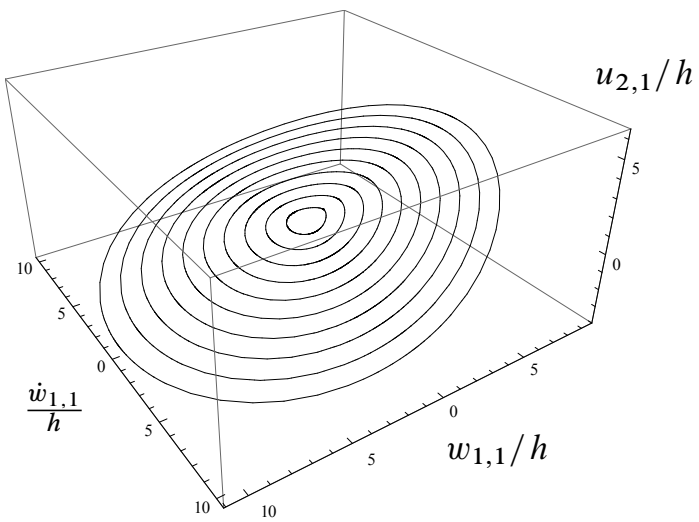

(b) $u_{2,1}$

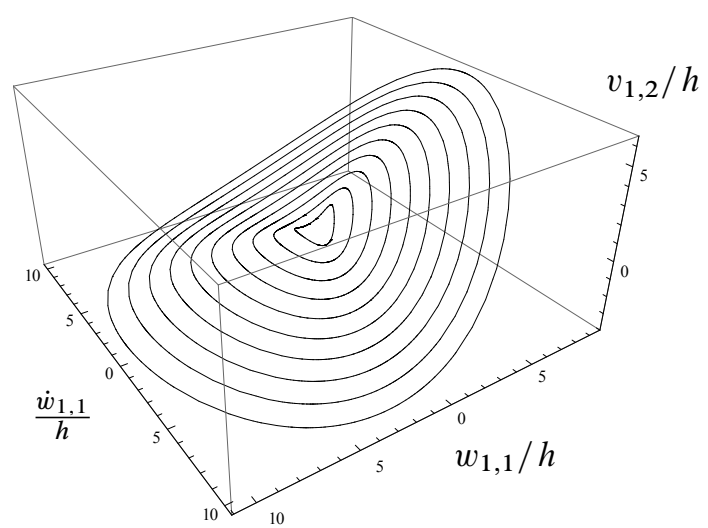

(d) $v_{1,2}$

Figure 10. Sections of the first non-linear modal manifold defined with respect to $w_{1,1}$ and $\dot{w}_{1,1}$

\section{Conclusions}

A method devoted to the analysis of large-amplitude vibrations of plates with physical as well as geometrical non-linearities is developed. The method is tested on a thin rectangular rubber plate. The problems of static bending under pressure and free and forced vibrations near the corresponding deformed configuration are investigated. Unlike most existing approaches, in the proposed method the sought shape of the deformed plate is not pre-assumed but is a direct output in the form of a series of linear modes.

A local model which describes the dynamics around a certain deformed configuration is systematically constructed. The advantage of this model is the simplicity. It is shown that the behavior of a rubber plate involving both geometrical and material non-linearities, being the non-linearity of higher order, can be accurately described by a system of ordinary differential equations with only quadratic and cubic polynomial terms. Static and dynamic responses approximated through the 
proposed technique compare well with the exact solutions. It is found that while at small strains the influence of the material non-linearity is weak, it becomes significant at finite strains. The deflection under a prescribed pressure is essentially underestimated when the material non-linearity is ignored.

Also, the vibration frequency in the vicinity of a deformed configuration is almost linear as opposed to the oscillations near the plate's flat configuration, which usually show strong amplitudefrequency dependence. However, strong non-linear effects like Neimark-Sacker bifurcations and internal resonances still emerge in the system: this justifies the necessity of non-linear models for the analysis of hyperelastic plates. As an extension, the developed method is suitable to thin-walled hyperelastic structures with more complicated geometries and more sophisticated hyperelastic laws.

\section{Acknowledgements}

The authors acknowledge the financial support of the NSERC Discovery Grant, the Canada Research Chair program of Canada, and the PSR-SIIRI program of Québec.

\section{A. Serial expansion of the transverse normal strain}

The coefficients involved in series (27) are detailed. Balancing the first power in the strain components yields the following system:

$$
\begin{aligned}
\lambda_{1}\left(\frac{1}{3(1-2 v)}+\frac{2}{3(1+v)}\right)+\frac{1}{3(1-2 v)}-\frac{1}{3(1+v)} & =0 \\
\lambda_{2}\left(\frac{1}{3(1-2 v)}+\frac{2}{3(1+v)}\right)+\frac{1}{3(1-2 v)}-\frac{1}{3(1+v)} & =0 \\
\lambda_{12}\left(\frac{1}{3(1-2 v)}+\frac{2}{3(1+v)}\right) & =0
\end{aligned}
$$

whose solution is:

$$
\lambda_{1}=\lambda_{2}=\frac{v}{v-1} \quad ; \quad \lambda_{12}=0
$$

Similarly, the set of equations for the coefficients of second power in the strain components is:

$$
\begin{aligned}
\lambda_{12,12}\left(\frac{1}{3(1-2 v)}+\frac{2}{3(1+v)}\right)-\frac{1}{6(1-2 v)} & =0 \\
\lambda_{2,12}\left(\frac{1}{3(1-2 v)}+\frac{2}{3(1+v)}\right) & =0 \\
\frac{1}{18}\left(\frac{3}{1-2 v}+\frac{4}{1+v}+\frac{6 \lambda_{2}}{1-2 v}+\frac{8 \lambda_{2}}{1+v}-\frac{9 \lambda_{2}^{2}}{1-2 v}-\frac{32 \lambda_{2}^{2}}{1+v}+\frac{6 \lambda_{2,2}}{1-2 v}+\frac{12 \lambda_{2,2}}{1+v}\right) & =0 \\
\lambda_{1,12}\left(\frac{1}{3(1-2 v)}+\frac{2}{3(1+v)}\right) & =0 \\
-\frac{2\left(-2-2 \lambda_{2}-2 \lambda_{1}+16 \lambda_{1} \lambda_{2}-3 \lambda_{1,2}\right)}{9(1+v)}+\frac{3+\lambda_{2}+\lambda_{1}-3 \lambda_{1} \lambda_{2}+\lambda_{1,2}}{3(1-2 v)} & =0 \\
\frac{1}{18}\left(\frac{3}{1-2 v}+\frac{4}{1+v}+\frac{6 \lambda_{1}}{1-2 v}+\frac{8 \lambda_{2}}{1+v}-\frac{9 \lambda_{1}^{2}}{1-2 v}-\frac{32 \lambda_{1}^{2}}{1+v}+\frac{6 \lambda_{1,1}}{1-2 v}+\frac{12 \lambda_{1,1}}{1+v}\right) & =0
\end{aligned}
$$

whose solution is:

$$
\begin{aligned}
& \lambda_{1,1}=\lambda_{2,2}=\frac{7-33 v+40 v^{3}}{18(v-1)^{3}} \quad ; \quad \lambda_{1,2}=\frac{(v+1)\left(13-52 v+46 v^{2}\right)}{9(v-1)^{3}} \\
& \lambda_{12,12}=\frac{v+1}{6(v-1)} \quad ; \quad \lambda_{1,12}=\lambda_{2,12}=0
\end{aligned}
$$


Linear systems of higher powers are omitted here for sake of brevity. Still, the coefficients of third power are:

$$
\begin{aligned}
& \lambda_{1,1,1}=\lambda_{2,2,2}=\frac{(v+1)\left(-62+274 v-123 v^{2}-560 v^{3}+520 v^{4}\right)}{81(v-1)^{5}} \\
& \lambda_{1,1,2}=\lambda_{1,2,2}=\frac{(v+1)\left(-46+94 v+349 v^{2}-1028 v^{3}+5680 v^{4}\right)}{27(v-1)^{5}} \\
& \lambda_{1,12,12}=\lambda_{2,12,12}=-\frac{(v+1)\left(4-37 v+40 v^{2}\right)}{27(v-1)^{3}} \\
& \lambda_{1,1,12}=\lambda_{2,2,12}=\lambda_{1,2,12}=\lambda_{12,12,12}=0
\end{aligned}
$$

and the coefficients of the fourth powers are:

$$
\begin{aligned}
& \lambda_{1,1,1,1}=\lambda_{2,2,2,2}=\frac{(v+1)\left(1436-4554 v-8547 v^{2}+39712 v^{3}-24012 v^{4}-32640 v^{5}+30320 v^{6}\right)}{1458(v-1)^{7}} \\
& \lambda_{1,1,1,2}=\lambda_{1,2,2,2}=\frac{2(v+1)\left(113+4536 v-27393 v^{2}+46102 v^{3}-279 v^{4}-61404 v^{5}+40040 v^{6}\right)}{729(v-1)^{7}} \\
& \lambda_{1,1,2,2}=\frac{(v+1)\left(-376+7086 v-30387 v^{2}+40840 v^{3}+15600 v^{4}-75216 v^{5}+44168 v^{6}\right)}{243(v-1)^{7}} \\
& \lambda_{1,1,12,12}=\lambda_{2,2,12,12}=-\frac{(v+1)\left(-147+716 v-515 v^{2}-1036 v^{3}+1080 v^{4}\right)}{729(v-1)^{5}} \\
& \lambda_{1,2,12,12}=-\frac{(v+1)\left(-155+620 v-151 v^{2}-1444 v^{3}+1228 v^{4}\right)}{81(v-1)^{5}} \\
& \lambda_{12,12,12,12}=\frac{(v+1)\left(-2-28 v+37 v^{2}\right)}{162(v-1)^{3}} \\
& \lambda_{1,1,1,12}=\lambda_{1,1,2,12}=\lambda_{1,2,2,12}=\lambda_{2,2,2,12}=\lambda_{1,12,12,12}=\lambda_{2,12,12,12}=0
\end{aligned}
$$

\section{B. Consistency between the neo-Hookean and the linear elasticity strain en- ergy densities}

Three-dimensional strain-stress relations lead to [3]:

$$
\epsilon_{3}=\frac{\sigma_{3}}{E}-\frac{v}{E}\left(\sigma_{1}+\sigma_{2}\right)
$$

Now we focus at expressions for infinitesimal strains. For thin plates we can assume $\sigma_{3} \cong 0$. Under this assumption,

$$
\sigma_{1}=\frac{E}{1-v^{2}}\left(\epsilon_{1}+v \epsilon_{2}\right) \quad ; \quad \sigma_{2}=\frac{E}{1-v^{2}}\left(\epsilon_{2}+v \epsilon_{1}\right)
$$

yielding:

$$
\epsilon_{3}=\frac{v}{v-1}\left(\epsilon_{1}+\epsilon_{2}\right)
$$

Then, the invariants (15) can be written as:

$$
\begin{aligned}
& I_{1}=2 \frac{2 v-1}{v-1}\left(\epsilon_{1}+\epsilon_{2}\right)+3 \\
& J^{2}=1+2 \frac{2 v-1}{v-1}\left(\epsilon_{1}+\epsilon_{2}\right)+\frac{4 v}{v-1}\left(\epsilon_{1}^{2}+\epsilon_{2}^{2}\right)+\frac{4(3 v-1)}{v-1} \epsilon_{1} \epsilon_{2}-\epsilon_{12}^{2}+O\left(\epsilon^{3}\right)
\end{aligned}
$$


The asymptotic expansion is applied to $J^{-\frac{2}{3}}$ (neglecting terms $O\left(\epsilon^{3}\right)$ ):

$$
\begin{aligned}
J^{-\frac{2}{3}} \approx 1- & \frac{1}{3}\left(2 \frac{2 v-1}{v-1}\left(\epsilon_{1}+\epsilon_{2}\right)+\frac{4 v}{v-1}\left(\epsilon_{1}^{2}+\epsilon_{2}^{2}\right)+\frac{4(3 v-1)}{v-1} \epsilon_{1} \epsilon_{2}-\epsilon_{12}^{2}\right) \\
& \quad+\frac{2}{9} \frac{4(2 v-1)^{2}}{(v-1)^{2}}\left(\epsilon_{1}^{2}+\epsilon_{2}^{2}+2 \epsilon_{1} \epsilon_{2}\right) \\
= & 1-\frac{2(2 v-1)}{3(v-1)}\left(\epsilon_{1}+\epsilon_{2}\right)+\frac{4\left(2-5 v+5 v^{2}\right)}{9(v-1)^{2}}\left(\epsilon_{1}^{2}+\epsilon_{2}^{2}\right)+\frac{4\left(1-4 v+7 v^{2}\right)}{9(v-1)^{2}} \epsilon_{1} \epsilon_{2}+\frac{1}{3} \epsilon_{12}^{2}
\end{aligned}
$$

The first term in (9) is transformed into:

$$
\begin{aligned}
\bar{I}_{1}-3 & =I_{1} J^{-\frac{2}{3}}-3=\left(2 \frac{2 v-1}{v-1}\left(\epsilon_{1}+\epsilon_{2}\right)+3\right) J^{-\frac{2}{3}}-3 \\
& \approx \frac{4\left(1-v+v^{2}\right)}{3(v-1)^{2}}\left(\epsilon_{1}^{2}+\epsilon_{2}^{2}\right)-\frac{4\left(1-4 v+v^{2}\right)}{3(v-1)^{2}} \epsilon_{1} \epsilon_{2}+\epsilon_{12}^{2}
\end{aligned}
$$

The second term in (9) has expansion:

$$
(J-1)^{2}=\left(\sqrt{J^{2}}-1\right)^{2} \approx \frac{1-4 v+4 v^{2}}{(v-1)^{2}}\left(\epsilon_{1}^{2}+\epsilon_{2}^{2}\right)+\frac{2(2 v-1)^{2}}{(v-1)^{2}} \epsilon_{1} \epsilon_{2}
$$

Expressions (62) and (63) are substituted into (9) and the coefficients of equal powers in $\epsilon_{i} \epsilon_{j}$, $i, j=1,2,12$ are collected. This gives:

$$
W=\frac{E\left(\bar{I}_{1}-3\right)}{4(1+v)}+\frac{E(J-1)^{2}}{6(1-2 v)} \approx E\left(\frac{\epsilon_{1}^{2}+\epsilon_{2}^{2}}{2\left(1-v^{2}\right)}+\frac{v \epsilon_{1} \epsilon_{2}}{1-v^{2}}+\frac{\epsilon_{12}^{2}}{4(1+v)}\right)
$$

and this expression coincides with Eq. (10).

\section{References}

[1] N. Akkas. "On the dynamic snap-out instability of inflated non-linear spherical membranes". International Journal of Non-Linear Mechanics 13(3) 1978, 177-183.

ISSN: 0020-7462.

DOI: 10 . 1016/0020-7462(78)90006-9.

[2] U. Akyüz and A. Ertepinar. "Stability and asymmetric vibrations of pressurized compressible hyperelastic cylindrical shells". International Journal of Non-Linear Mechanics 34(3) 1998, 391-404.

ISSN: 0020-7462.

DOI: $10.1016 / \mathrm{S} 0020-7462$ (98)00015-8.

[3] M. Amabili. Nonlinear vibrations and stability of shells and plates. New York: Cambridge University Press, 2008. ISBN: 9780521883290.

[4] M. Amabili. "Nonlinear vibrations of rectangular plates with different boundary conditions: theory and experiments". Computers and Structures 82(31-32) 2004, 2587-2605.

ISSN: 0045-7949.

DOI: $10.1016 / j$.compstruc. 2004.03 .077$.

[5] M. Amabili. "Theory and experiments for large-amplitude vibrations of empty and fluid-filled circular cylindrical shells with imperfections". Journal of Sound and Vibration 262(4) 2003, 921-975. ISSN: 0022-460X.

DOI: $10.1016 / \mathrm{S} 0022-460 \mathrm{X}$ (02)01051-9.

[6] J. Awrejcewicz and V. Krys'ko. Nonclassical thermoelastic problems in nonlinear dynamics of shells. Scientific Computation. Berlin: Springer, 2003. ISBN: 9783540438809.

[7] A. Bower. Applied mechanics of solids. Boca Raton: CRC Press. Taylor and Francis Group, 2010.

[8] I. Breslavsky. "Stress distribution over plates vibrating at large amplitudes". Journal of Sound and Vibration 331(12) 2012, 2901-2910.

DOI: $10.1016 / j \cdot j$. sv . 2012.02.002. 
[9] I. Breslavsky and K. Avramov. "Two modes nonresonant interaction for rectangular plate with geometrical nonlinearity". Nonlinear Dynamics 69(1-2) 2012, 285-294.

ISSN: 0924-090X. DOI: $10.1007 / \mathrm{s} 11071-011-0264-3$.

[10] C. Calderer. "The dynamical behaviour of nonlinear elastic spherical shells". Journal of Elasticity 13(1) 1983, 17-47. ISSN: 0374-3535. DOI: $10.1007 / \mathrm{BF} 00041312$.

[11] C.Y. Chia. Non-linear analysis of plates. New York: McGraw-Hill, 1980.

[12] E. Doedel, A. Champneys, T. Fairgrieve, Y. Kuznetsov, B. Sandstede, and X. Wang. AUTO 97: Continuation and Bifurcation Software for Ordinary Differential Equations (with HomCont). Montreal: Concordia University, 1998.

[13] J. Fox and N. Goulbourne. "Electric field-induced surface transformations and experimental dynamic characteristics of dielectric elastomer membranes". Journal of the Mechanics and Physics of Solids 57(8) 2009, 1417-1435.

ISSN: 0022-5096.

DOI: $10.1016 / j \cdot j m p s .2009 .03 .008$.

[14] J. Fox and N. Goulbourne. "On the dynamic electromechanical loading of dielectric elastomer membranes". Journal of the Mechanics and Physics of Solids 56(8) 2008, 2669-2686. ISSN: 0022-5096. DOI: $10.1016 / j \cdot j m p s .2008 .03 .007$.

[15] Y. Fu and R. Ogden. Nonlinear Elasticity: Theory and applications. Cambridge: Cambridge University Press, 2001. ISBN: 9780521796958. DOI: $10.1017 / \mathrm{CB} 09780511526466$.

[16] P. Goncalves, R. Soares, and D. Pamplona. "Nonlinear vibrations of a radially stretched circular hyperelastic membrane". Journal of Sound and Vibration 327(1-2) 2009, 231-248. ISSN: 0022-460X. DOI: $10.1016 / j \cdot j$.j. 2009.06.023.

[17] H. Haslach and J. Humphrey. "Dynamics of biological soft tissue and rubber: internally pressurized spherical membranes surrounded by a fluid". International Journal of Non-Linear Mechanics 39(3) 2004, 399-420. ISSN: 0020-7462. DOI: $10.1016 / \mathrm{S} 0020-7462(02) 00196-8$.

[18] Y. Ju and D. Niu. "On a class of differential equations of motion of hyperelastic spherical membranes". Applied Mathematical Sciences 6(81-84) 2012, 4133-4136.

[19] L. Kachanov. Fundamentals of theory of plasticity. Mineola: Courier Dover Publications, 2004. ISBN: 9780486435831.

[20] J. Knowles. "Large amplitude oscillations of a tube of incompressible elastic material". Quarterly of Applied Mathematics 18 1960, 71-77.

[21] J. Knowles. "On a class of oscillations in the finite-deformation theory of elasticity". Journal of Applied Mechanics 29(2) 1962, 283-286. DOI: $10.1115 / 1.3640542$.

[22] A. Leissa. Vibration of plates. Washington, D.C.: Scientific, Technical Information Division, National Aeronautics, and Space Administration, 1969.

[23] R. Ogden. Non-Linear Elastic Deformations. Dover Civil and Mechanical Engineering Series. New York: Dover Publications, 1997. ISBN: 9780486696485.

[24] R. Ogden and D. Roxburgh. "The effect of pre-stress on the vibration and stability of elastic plates". International Journal of Engineering Science 31(12) 1993, 1611-1639. ISSN: 0020-7225. DOI: $10.1016 / 0020-7225$ (93) 90079-A.

[25] T. Parker and L. Chua. Practical numerical algorithms for chaotic systems. New York: Springer-Verlag, 1989. 
[26] M. Qatu. "Recent research advances in the dynamic behavior of shells: 1989-2000, Part 1: Laminated composite shells". Applied Mechanics Reviews 55(4) 2002, 325-350. DOI: $10.1115 / 1.1483079$.

[27] M. Qatu. "Recent research advances in the dynamic behavior of shells: 1989-2000, Part 2: Homogeneous shells". Applied Mechanics Reviews 55(5) 2002, 415-434. DOI: $10.1115 / 1.1483078$.

[28] J.-S. Ren. "Dynamical response of hyper-elastic cylindrical shells under periodic load". Applied Mathematics and Mechanics 29(10) 2008, 1319-1327. ISSN: 0253-4827. DOI: $10.1007 / \mathrm{s} 10483-008-1007-\mathrm{x}$.

[29] D. Roxburgh and R. Ogden. "Stability and vibration of pre-stressed compressible elastic plates". International Journal of Engineering Science 32(3) 1994, 427-454. ISSN: 0020-7225. DOI: $10.1016 / 0020-7225$ (94)90133-3.

[30] R. Soares and P. Goncalves. "Nonlinear vibrations and instabilities of a stretched hyperelastic annular membrane". International Journal of Solids and Structures 49(3-4) 2012, 514-526. ISSN: 0020-7683. DOI: $10.1016 / j$.ijsolstr.2011.10.019.

[31] E. Verron, R. Khayat, A. Derdouri, and B. Peseux. "Dynamic inflation of hyperelastic spherical membranes". Journal of Rheology 43(5) 1999, 1083-1097. DOI: $10.1122 / 1.551017$.

[32] V. Yakubovich and V. Starzhinskii. Linear differential equations with periodic coefficients. New York: Wiley, 1975.

[33] H. Yong, X. He, and Y. Zhou. "Dynamics of a thick-walled dielectric elastomer spherical shell". International Journal of Engineering Science 49(8) 2011, 792-800. ISSN: 0020-7225. DOI: $10.1016 / j$.ijengsci.2011.03.006.

[34] X. Yuan, H. Zhang, J. Ren, and Z. Zhu. "Some qualitative properties of incompressible hyperelastic spherical membranes under dynamic loads". Applied Mathematics and Mechanics 31(7) 2010, 903-910. ISSN: 0253-4827. DOI: $10.1007 / \mathrm{s} 10483-010-1324-6$.

[35] X. Yuan, R. Zhang, and H. Zhang. "Controllability conditions of finite oscillations of hyper-elastic cylindrical tubes composed of a class of Ogden material models". Computers, Materials and Continua 7 2008, 155-166. DOI: $10.3970 / \mathrm{cmc} .2008 .007 .155$.

[36] J. Zhu, S. Cai, and Z. Suo. "Nonlinear oscillation of a dielectric elastomer balloon". Polymer International 59(3) 2010, 378-383. DOI: $10.1002 / p i .2767$.

[37] J. Zhu, S. Cai, and Z. Suo. "Resonant behavior of a membrane of a dielectric elastomer". International Journal of Solids and Structures 47(24) 2010, 3254-3262. ISSN: 0020-7683. DOI: $10.1016 /$ j.ijsolstr.2010.08.008. 